\title{
LAS EXPULSIONES DE INDOCUMENTADOS MEXICANOS
}

\author{
Roberto Ham Chande \\ Jorge A. Bustamante \\ El Colegio de México
}

\section{INTRODUCCIÓN}

UNA DE LAS CARACTERí́sticas del espectro demográfico del presente siglo es la rapidez con la que han sobrevenido cambios. Estos cambios demográficos del mundo actual se presentan, además, con una complejidad sin precedente. Es ya clásica en la historia demográfica del siglo xx la observación de que el avance acelerado de la tecnología trajo como producto un abatimiento de la mortalidad a nivel mundial, aunque con variaciones contrastantes. Otros fenómenos poblacionales de este siglo se presentan con manifestaciones cuyo entendimiento es cada vez más difícil y su solución cada vez más urgente. Uno de estos fenómenos lo constituyen los movimientos migratorios.

A diferencia de otras componentes demográficas como son las de la fecundidad y la mortalidad, en las cuales se observan limitaciones naturales e inercias que admiten considerable libertad de medición de los fenómenos y su parametrización, no sucede así en el caso de la migración. Los movimientos migratorios se manifiestan fuera de delimitaciones naturales que permitan un mejor cotejo numérico de ellos y se sujetan a fluctuaciones de mayor carácter errático. La razón de la dificultad estriba en el hecho de que las corrientes migratorias tienen como motivo ingredientes de índole socioeconómico, con toda la complejidad que esto implica. Baste recordar que una migración puede ser una decisión individual, o familiar, o darse de manera masiva; puede ser en busca de una mejor oportunidad económica o puede ser producto de una guerra o de una persecusión política o religiosa. Tales peculiaridades presentan una dificultad inmediata cuando se intenta el estudio del fenómeno: la carencia de información estadística y la dificultad para generar datos.

Dentro de las diversas modalidades que revisten los fenómenos migratorios entre México y los Estados Unidos, existe una que es de importancia primordial para los dos países y que es la corriente migratoria de trabajadores indocumentados. ${ }^{1}$ A las dificultades propias de obtención y

1 El término "indocumentado" se refiere a la persona que emigra temporal o indefinidamente a los Estados Unidos sin la documentación migratoria correspondiente. 
manejo de datos estadísticos sobre migración, en el caso de la migración indocumentada se agrega el agravante de su índole subrepticia. Esto tiene como evidente resultado el que no exista notificación alguna, ni al país emisor ni al país receptor, de semejantes movimientos. En estas condiciones, encontramos que, para uno de los fenómenos demográficos de mayor importancia para México y los Estados Unidos, en el cual participan posiblemente millones de gentes en las edades de mayor producción y reproducción, carecemos de información numérica confiable ${ }^{2}$ que sirva de base en la búsqueda de soluciones conjuntas a los obvios problemas y tensiones que tal situación genera en ambos lados de la frontera.

La única fuente de información estadística sistematizada disponible se concentra en una sola de las facetas del fenómeno de la migración indocumentada y ésta se refiere a la captura y expulsión de este tipo de migrantes por parte de las autoridades norteamericanas. Cada vez que un indocumentado es detectado y capturado por el Servicio de Inmigración y Naturalización (SIN), ${ }^{3}$ del gobierno federal de los Estados Unidos, se registran una serie de datos que el sin compila mensualmente en forma de estadísticas sobre las nacionalidades y orígenes de los indocumentados capturados y bajo diversas clasificaciones de interés. Las cifras se presentan en las formas tituladas "Monthly Report of Deportable Aliens Found in the United States by Nationality, Status of Entry, Place of Entry, Status when Found". U. S. Department of Justice, Inmigration and Naturalization Service, Form G-23.18. En este trabajo se presenta un análisis puramente descriptivo de esas cifras mensuales de enero de 1972 a noviembre de 1977, con excepción del mes de marzo de 1973 cuyos datos no estuvieron disponibles, y en particular sobre las cifras referentes a la captura y expulsión de indocumentados de origen mexicano. ${ }^{4}$

\section{CARÁcter estadístico de la INFORMACión}

Las estadísticas sobre aprehensiones de inmigrantes indocumentados captadas por el sIN, constituyen una fuente de conocimiento que debe considerarse con algunas limitaciones que la información trae consigo.

2 El Centro Nacional de Información y Estadísticas del Trabajo (CENIET) está realizando un proyecto de investigación con base en una encuesta nacional cuyo objetivo principal es obtener esta información de la que hasta ahora se carece. Véase, Jerónimo Martínez, "Encuesta nacional de emigración a la frontera norte del país y a los Estados Unidos; descripción del proyecto y hallazgos de la $2^{a}$ etapa (agosto de 1978)", ponencia presentada en el Simposio sobre Estudios Fronterizos en Monterrey, N. L. en enero de 1979.

3 El Servicio de Inmigración y Naturalización se denomina oficialmente como U. S. Immigration and Naturalization Service.

4 Aunque los informes se refieran a extranjeros "deportables" no se trata precisamente de deportaciones. Una deportación requiere de un procedimiento legal que por lo general no se da en esta clase de expulsiones. 
Estas limitaciones son ante todo respecto a la representatividad de las cifras. Se debe definir con toda exactitud lo que expresan esas cifras, para lo cual es necesario señalar dos características de esas estadísticas. En primer lugar, las cantidades sobre aprehendidos en realidad reflejan el número de eventos de aprehensión y no el de individuos que fallan en su propósito de emigrar como indocumentados, al ser capturados. Un mismo individuo pudo ser detenido varias veces en el mismo periodo de referencia de las estadísticas, como en realidad sucede con gran frecuencia. En segundo término, y tal y como se explica con mayor detalle después, las aprehensiones no se hacen puramente al azar, responden a una cierta política selectiva de detenciones que puede marcar una acción más intensiva en regiones determinadas de los Estados Unidos, como es el caso de la frontera con México, lo cual genera una desproporción en el número de mexicanos detectados respecto a la proporción de indocumentados mexicanos que realmente existe. Otro ejemplo de esto último lo representa la práctica que el sin a veces sigue de intensificar su acción de vigilancia en ciertas industrias, lo cual implica un aumento en las cifras sobre expulsiones en el periodo de la acción del SIN sobre trabajadores indocumentados. De esta manera, se imposibilita la estimación estadística de dos aspectos importantes para el estudio de este fenómeno a partir de las cifras de las aprehensiones y que son, por una parte, el volumen de la inmigración indocumentada y, por otra, las características de los indocumentados.

No obstante las restricciones señaladas, se considera conveniente estudiar las estadísticas de las aprehensiones, pues en ellas se ve reflejada una parte del fenómeno demográfico de la migración indocumentada, que es la captura misma y la devolución de esos mexicanos al territorio nacional, en un proceso que esencialmente se da en la frontera y cuyas características y consecuencias no han sido todavía estudiadas lo suficiente.

Desde otro punto de vista, analizar estas estadísticas servirá como una indicación del tipo de capacidad de detención que tienen las autoridades migratorias norteamericanas y de la clase de estrategia seguida en esta actividad. Se han hecho cargos de que la fuerza policial del sIN no funciona de modo constante a toda su capacidad, sino que, en última instancia, responde a las solicitudes de los empresarios norteamericanos para relajar o fortalecer las detenciones de acuerdo con sus necesidades de mano de obra. ${ }^{5}$ En todo caso, se puede conjeturar que las estadísticas de aprehensiones del sIN reflejan una combinación de rasgos derivados en parte por la variación en la selectividad de las aprehensiones, en parte por la posible exclusión de indocumentados de ciertas caracteris-

5 Véase Jorge A. Bustamante, "El delito de ser espalda mojada", en Anovar Abdel-Malek (Comp.), Sociología del imperialismo, México, Instituto de Investigaciones Sociales, UNAM, 1977, pp. 345-360. 
ticas y, en parte también, por las variaciones en la conducta de los propios indocumentados detenidos y expulsados.

A pesar de estas advertencias, se encontró ventajoso el hecho de que el sin recabe todos los datos de las detenciones de indocumentados y los tabule mes por mes. Además, si se dispone de estas tabulaciones mensuales para un periodo de varios años, es posible hacer un análisis cronológico, efectuar comparaciones en periodos semejantes y buscar pautas de recurrencia.

\section{SELECCIÓN Y PRESENTACIÓN DE LA INFORMACIÓN}

De toda la información que contienen las tabulaciones sobre "extranjeros ilegales" detectados por las autoridades de migración de los Estados Unidos, se han seleccionado aquellas partes correspondientes a los indocumentados mexicanos, tal y como se expresó en la introducción. Estos datos se encuentran agrupados en cuatro clases de variables: a) sexo; b) tiempo de estancia; c) situación migratoria y; d) condición laboral.

En la clasificación respecto al sexo no se hace una dicotomía estricta entre hombres y mujeres; se agrupa en la primera categoría a los hombres adultos y en la segunda a las mujeres y los niños.

El tiempo de estancia en los Estados Unidos se refiere al tiempo transcurrido desde el momento de su arribo a territorio norteamericano como indocumentados hasta el momento de su captura. La información sobre esta variable adopta la siguiente categorización: a) capturados al momento de la entrada; b) capturados en las primeras 72 horas; $c$ ) capturados entre cuatro y 30 días de su entrada; d) capturados de uno a seis meses después de su llegada; $e$ ) capturados entre siete meses y un año después de su arribo y; $f$ ) capturados después de un año de estancia.

Sobre su situación migratoria, la categorización tiene cinco partes: a) visitantes; b) estudiantes; c) inmigrantes; $d$ ) personas que se introdujeron sin ser inspeccionadas $\mathrm{y} ; e$ ) otros.

La condición laboral de los capturados considera también cinco categorías: $a$ ) en la agricultura; $b$ ) en la industria y otros; $c$ ) en busca de empleo; $d$ ) en instituciones y; $e$ ) viajando.

Los cuadros estadísticos que se muestran a lo largo de este trabajo son una selección y elaboración numérica de las cifras compiladas por el SIN. Las cifras que se citan de manera directa no tienen el detalle con las que el SIN las desglosa, y han sido resumidas para efectos de presentación en este trabajo como ilustración de aquellos aspectos que se desea destacar.

El procedimiento seguido para el análisis que aquí se presenta se inició con la transcripción a un archivo magnético de toda la información correspondiente a indocumentados mexicanos, tomada de las formas 
G-23.18 del sin. Una vez depurado el archivo, se produjeron por computación e impresión electrónica una serie de gráficas a partir del "cruce" de cifras de indocumentados capturados, por meses durante los años de 1972 a $1977 .^{6}$ Se hizo una selección de las gráficas más ricas en información respecto a ciertas recurrencias y situaciones sobresalientes que se comentarán más adelante.

En la lectura de este artículo se hace necesario también una advertencia sobre las cifras utilizadas. Gracias al auxilio de las herramientas de computación se pudieron producir un gran número de tabulaciones y estimaciones estadísticas, algunas de las cuales se transcriben en forma de cuadros dentro del mismo trabajo. En otras ocasiones sólo se citan algunos de los resultados obtenidos, sin recurrir a su transcripción en cuadros dentro del artículo para no recargarlo con cifras.

\section{El MOVIMIENTO GENERAL DE LAS EXPULSIONES}

Con base en cifras estimadas para los meses de marzo de 1973 y diciembre de 1977 respecto de los cuales no se disponía de información, ${ }^{7}$ se observa que entre los años de 1972 y 1977 el sIN realizó un total de

\section{Cuadro 1}

TOTAL DE EXPULSIONES DE MEXICANOS POR AÑo

\begin{tabular}{lc}
\hline A x́o & Número de expulsiones \\
\hline 1972 & 491821 a \\
1973 & 627880 I \\
1974 & 718161 \\
1975 & 692902 \\
1976 & $789907 \mathrm{~b}$ \\
1977 & 993861 \\
Total & 4314532 \\
\hline
\end{tabular}

a Incluye las expulsiones estimadas para el mes de marzo.

b Incluye las expulsiones estimadas para el mes de diciembre.

4314532 expulsiones de mexicanos. Estas expulsiones se distribuyen anualmente de la manera señalada en el cuadro 1.

La información del cuadro 1 muestra el carácter ascendente del fenómeno. En la serie de datos, el único año que no registra la tendencia de

6 En todo el trabajo correspondiente al archivo magnético y la programación posterior de elementos estadísticos y gráficas, contamos con la muy eficiente colaboración de Patricia Fernández.

7 Las estimaciones se hicieron a través de la observación de los porcentajes que representan los meses faltantes del total de expulsiones en aquellos años en los que sí se contó con el dato. 
crecimiento es 1975 , donde se observa una ligera disminución respecto al año anterior. Después de tal fecha la tendencia se mantiene. Entre 1972 y 1973 el aumento medio anual de las expulsiones es de 83673 . Conforme a un modelo de regresión lineal que se utilizó para ajustar el número de expulsiones respecto a cada año, se encontró como medida de ese crecimiento anual un coeficiente de 84800 . (El ajuste de los datos mediante un modelo tan simple resultó bastante preciso pues se observa un coeficiente de correlación de 0.9451.)

En la interpretación de las cifras y gráficas presentadas en este trabajo, se debe tener en cuenta que éstas reflejan el comportamiento de las dos partes involucradas en las aprehensiones del sIN. Por un lado se tiene el comportamiento y las prácticas de los migrantes indocumentados, y por otro la actuación de las autoridades norteamericanas respecto a aprehensiones y expulsiones. El estado de la información no permite dilucidar en qué medida interviene cada factor o cuál es el peso de su interrelación. De esta manera, las variaciones en las cifras mensuales resultan de la mezcla de ambos tipos de factores, sin que pueda distinguirse en qué proporción interviene cada uno de ellos.

En la gráfica 1 se observa con claridad la tendencia creciente del número de expulsiones registradas. En la misma gráfica, además de los cambios en el número total de expulsiones, se presentan los cambios registrados en las categorías de "hombres" y "mujeres y niños", que muestran también la misma tendencia al aumento. Otra caracteristica del fenómeno es la existencia de una norma de recurrencia cíclica por años.

\section{Cuadro 2}

EXPULSIONES TOTALES DE MEXICANOS POR MES Y AÑO, $Y$ PORCENTAJE SEGÚN EL MES

\begin{tabular}{lrrrrrrrrr}
\hline & 1972 & 1973 & 1974 & 1975 & 9176 & 1977 & af \\
\hline Enero & 27988 & 36222 & 52170 & 45608 & 58780 & 61804 & 6.73 \\
Febrexo & 29373 & 42728 & 59224 & 49174 & 61556 & 65265 & 7.32 \\
Marzo & 41722 & & - & 76130 & 68019 & 79396 & 88341 & 8.44 \\
Abril & 44608 & 56366 & 72870 & 61273 & 77833 & 85551 & 9.50 \\
Mayo & 49872 & 63983 & 74440 & 67093 & 84500 & 104742 & 10.60 \\
Junio & 43225 & 60553 & 68929 & 63264 & 80901 & 109625 & 10.16 \\
Julio & 47484 & 52533 & 66805 & 59033 & 74543 & 101764 & 9.58 \\
Agosto & 50 & 163 & 57822 & 65247 & 64560 & 64375 & 99469 & 9.57 \\
Septiembre & 49048 & 59545 & 58222 & 62030 & 59635 & 89514 & 9.01 \\
Octubre & 47051 & 54545 & 47544 & 58654 & 55280 & 71821 & 7.98 \\
Noviembre & 34571 & 45327 & 41613 & 47966 & 48533 & 59212 & 6.61 \\
Diciembre & 26716 & 36410 & 34967 & 46228 & 44575 & & - & 4.50 \\
\hline
\end{tabular}

a Este porciento representa la proporción de capturas totales que corresponde a cada mes (suma horizontal) respecto al total de aprehensiones realizadas durante los seis años considerados. 
Gráfica 1

DEPORTACIONES DE INDOCUMENTADOS MEXICANOS

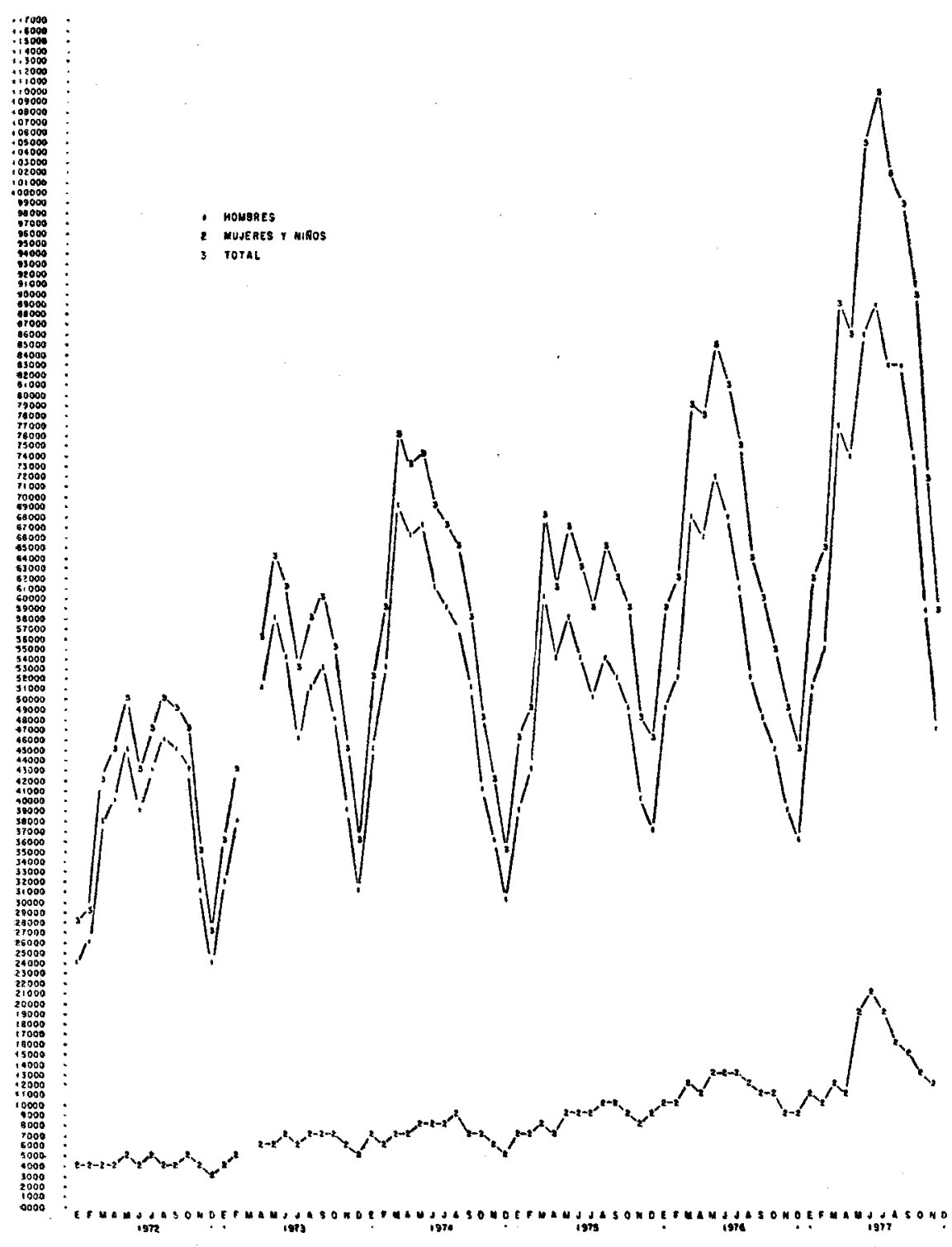


Esta estacionalidad se caracteriza por un nivel máximo de expulsiones durante los meses de verano y uno mínimo en los de invierno.

Es notable el carácter cíclico a lo largo de toda la serie, lo cual sugiere una característica de temporalidad de la migración indocumentada, con gran probabilidad debida a variaciones estacionales en la conducta migratoria. Los ciclos registran volúmenes importantes de expulsiones a lo largo de los ocho meses consecutivos que van de marzo a octubre, y un descenso sólo en lo que resta del año, o sea en los meses de noviembre a febrero.

El movimiento cíclico se manifiesta también en el cuadro 2, donde se presentan las cifras del total de expulsiones en cada mes durante los

\section{Cuadro 3}

ÍNDICE DE VARIACIÓN CÍCLICA MENSUAL DE LAS EXPULSIONES DE MEXICANOS

\begin{tabular}{lcccccccr}
\hline & 1972 & 1973 & 1974 & 1975 & 1976 & 1977 & Hedia, & cval \\
\hline Enero & 0.7135 & 0.7829 & 0.9785 & 0.7556 & 0.8720 & 0.8301 & 0.8221 & 10.51 \\
Febrero & 0.7378 & 0.9119 & 1.0987 & 0.8068 & 0.9053 & 0.8698 & 0.8884 & 12.56 \\
Ifarzo & 1.0328 & & $\mathbf{1 . 3 9 7 2}$ & 1.1054 & 1.1577 & 1.1748 & 1.1735 & 10.42 \\
Abri1 & 1.0884 & 1.1735 & 1.3231 & 0.9863 & 1.1253 & 1.1225 & 1.1365 & 8.90 \\
Mayo & 1.1996 & 1.3160 & 1.3373 & 1.0699 & 1.2114 & 1.3638 & 1.2497 & 8.10 \\
Junio & 1.0253 & 1.2306 & 1.2254 & 0.9995 & 1.1501 & 1.4165 & 1.1746 & 11.93 \\
Julio & 1.1108 & 1.0551 & 1.1754 & 0.9241 & 1.0510 & 1.3051 & 1.1036 & 10.67 \\
Agosto & 1.1576 & 1.1477 & 1.1362 & 1.0014 & 0.9002 & 1.2651 & 1.1015 & 10.75 \\
Septiembre & 1.1167 & 1.1638 & 1.0016 & 0.9535 & 0.8271 & 1.1310 & 1.0334 & 11.49 \\
Octubre & 1.0571 & 1.0580 & 0.8113 & 0.8935 & 0.7605 & 0.9007 & 0.9135 & 12.32 \\
Noviembre & 0.7666 & 0.8993 & 0.7031 & 0.7242 & 0.6623 & 0.7372 & 0.7438 & 8.67 \\
Diciembre & 0.5848 & 0.6905 & 0.5850 & 0.6918 & 0.6035 & & 0.6311 & 7.84 \\
Media & 0.9659 & 1.0390 & 1.0646 & 0.9093 & 0.9355 & 1.1016 & & \\
\hline
\end{tabular}

años observados. En la última columna de este cuadro se expresan las proporciones de aprehensiones durante cada mes respecto del total. La manera en que se distribuyen dichas proporciones indica la mayor incidencia en los meses de verano y la menor en los de invierno.

Con objeto de determinar numéricamente la estacionalidad conforme a la tendencia al crecimiento, se construyeron ciertos "índices" para todas las series cronológicas que determinaron el movimiento cíclico. Estos índices se definen para cada observación mensual como el número de veces que tiene lugar dicha observación, respecto a su estimación, bajo un modelo de regresión lineal simple. Para el caso de las estadísticas mensuales sobre el total de expulsiones se obtiene una recta de regresión alrededor de la cual se registran los índices de acuerdo a la definición dada para ellos (véase el cuadro 3 ).

Tales índices tienen el siguiente significado. Si el índice adquiere exactamente el valor de 1, quiere decir que el número de expulsiones en tal caso corresponden a lo estimado por el modelo lineal. Si el índice es mayor que 1 las observaciones están por encima del valor lineal, y en 
el caso de que se den valores menores que 1 se trata de datos por debajo de esos valores lineales.

Junto a los índices para cada mes de cada año, se calculó la media observada en cada mes calendario para todo el periodo, así como el coeficiente de variación ${ }^{8}$ (cv) resultante. Se observa en esos valores medios una gran coincidencia con el resto de los índices año con año, hecho que se denota por los reducidos valores de los coeficientes de variación. De esta manera, se manifiesta el carácter cíclico anual mencionado, con valores mínimos en la época invernal (cifras menores y regularmente más chicas que 1), y valores por encima de 1 , también en forma regular, con índices máximos en los meses de mayo, junio y julio.

En la gráfica 1 se puede observar un descenso en el año de 1975, en particular en los meses de mayores niveles de aprehensiones. En los meses de mayor actividad en las expulsiones (con índices medios mayores que 1), de marzo a septiembre, para 1975 se observan valores en general menores y en la mayoría de los casos incluso menores que 1. Este hecho se manifiesta cuando se observan los valores medios por año de los índices, en los cuales justamente 1975 tiene el valor de 0.9093, el menor de todos.

Este descenso de 1975 coincide con un aumento crítico en los niveles de desempleo en los Estados Unidos. ${ }^{9}$ Esta coincidencia sugiere una correspondencia entre el aumento del desempleo en aquel país y la disminución del volumen de inmigrantes indocumentados hacia la frontera norte, y por lo tanto de las detenciones. Si las cifras reflejan tal comportamiento, podría sugerirse la hipótesis de que la disminución en el volumen del movimiento migratorio indocumentado en 1975 fue consecuencia directa de la disminución de oportunidades de trabajo que en términos generales se dio en ese año en los Estados Unidos. ${ }^{10}$ De ser cierta esta hipótesis se podría concluir que la emigración de indocumentados. de México a los Estados Unidos responde de manera muy sensible a las condiciones de la demanda de mano de obra en los Estados Unidos y no sólo a las condiciones llamadas de expulsión que se producen en México, de las cuales se deriva la oferta de mano de obra de indocumentados.

Al examinar el comportamiento separado entre "hombres" y "mujeres y niños" (véase la gráfica 1), se aprecia que para cada categoría se repite la tendencia creciente. También en el caso de "hombres" se manifiesta un movimiento cíclico. Sin embargo, no lo es tanto para "mujeres

8 El coeficiente de variación es el cociente de la desviación estándar entre la media, multiplicado por 100 . Expresa, en términos porcentuales respecto a la media, el grado de variación que hay entre las cifras.

9 U. S. National Commission for Manpower Policy, Manpower and Immigration Policies in the United States, Informe Especial No 20 (febrero de 1978), Washington, D. C.: Imprenta del Gobierno de los Estados Unidos, 1978, pp. 174-175.

10 Ibidem. 
y niños", lo cual se confirma al examinarse los índices medios y sus coeficientes de variación mes tras mes, tal y como se observa en el cuadro 4.

El índice que corresponde a la clasificación "hombres" denota de manera evidente el carácter cíclico del fenómeno, con coeficientes de variación ligeramente mayores que para el caso general. Para "mujeres y niños" también existe cierta estacionalidad manifestada a través de los valores de los índices; sin embargo, su amplitud es menos marcada, como lo denotan los mismos índices y su mayor coeficiente de variación.

\section{Cuadro 4}

INDICE MEDIO DE ESTACIONALIDAD MENSUAL Y SU COEFICIENTE DE VARIACIÓN ( $C V$ ) PARA "HOMBRES" $Y$ "MUJERES $Y$ NIÑOS"

\begin{tabular}{|c|c|c|c|c|}
\hline & \multicolumn{2}{|c|}{ Homb res } & \multicolumn{2}{|c|}{ Nujeres y nifios } \\
\hline & Indice & $c v$ & Indice & $c v$ \\
\hline $\begin{array}{l}\text { Enero } \\
\text { Febrero } \\
\text { Marzo } \\
\text { Mayo } \\
\text { Junio } \\
\text { Julio } \\
\text { Agosto } \\
\text { Septiembre } \\
\text { Octubre } \\
\text { Noviembre } \\
\text { Diciembre }\end{array}$ & $\begin{array}{l}0.8054 \\
0.8883 \\
1.2017 \\
1.2588 \\
1.1730 \\
1.0967 \\
1.0001 \\
1.0327 \\
0.9057 \\
0.7294 \\
0.6135\end{array}$ & $\begin{array}{r}12.44 \\
14.31 \\
12.14 \\
8.56 \\
11.68 \\
11.90 \\
11.94 \\
12.53 \\
13.15 \\
8.81 \\
7.18\end{array}$ & $\begin{array}{l}0.9978 \\
0.9306 \\
1.0395 \\
1.1997 \\
1.1854 \\
1.1457 \\
1.1080 \\
1.0269 \\
0.9551 \\
0.8290 \\
0.7388\end{array}$ & $\begin{array}{r}25.55 \\
16.20 \\
14.64 \\
14.26 \\
15.29 \\
11.55 \\
5.04 \\
7.49 \\
8.62 \\
10.75 \\
10.83\end{array}$ \\
\hline
\end{tabular}

En ambas categorías, se observa que en 1975 se verifica el mismo fenómeno de disminución de expulsiones. El valor medio de los índices es de 0.9111 para "hombres" y de 0.8973 para "mujeres y niños", los más bajos registrados de cada año.

\section{EXPULSIONES DE ACUERDO CON EL TIEMPO DE ESTANCIA}

En las gráficas 2, 3 y 4 se expresan los movimientos de expulsión de indocumentados de acuerdo con el tiempo de estancia en los Estados Unidos, desde su arribo a territorio norteamericano hasta su captura. La gráfica 2 representa el panorama global de las expulsiones de acuerdo con los seis lapsos de estancia definidos antes. Esta gráfica se refiere a las cifras sobre "hombres adultos", en tanto que la gráfica 4 muestra los datos referentes a la categoría de "mujeres y niños".

Conforme a la gráfica 2, se observa que del total de expulsiones, en función del tiempo de estancia, en general existe una misma pauta en los cambios cíclicos anuales y que éstos son más notorios conforme el tiempo de estancia es más corto. Es claro en particular que los capturados en la primera categoría, o sea los capturados prácticamente al momento 
de su entrada a los Estados Unidos, son los que representan la categoría de mayor volumen. A lo largo de los seis años considerados, los capturados al momento de su entrada han representado el $39.01 \%$ del total de capturas. Un elemento adicional de distinción en esta categoría es que tiene una mayor tendencia al incremento. Su coeficiente de crecimiento en la recta de regresión lineal es de 397.15, en comparación, por ejemplo, con el siguiente de mayor valor (62.99), correspondiente a los capturados después de 24 horas de su llegada y antes de las 72 horas. Ambas

\section{Gráfica 2}

Deportaciones según tiempo de estancia hasta la captura

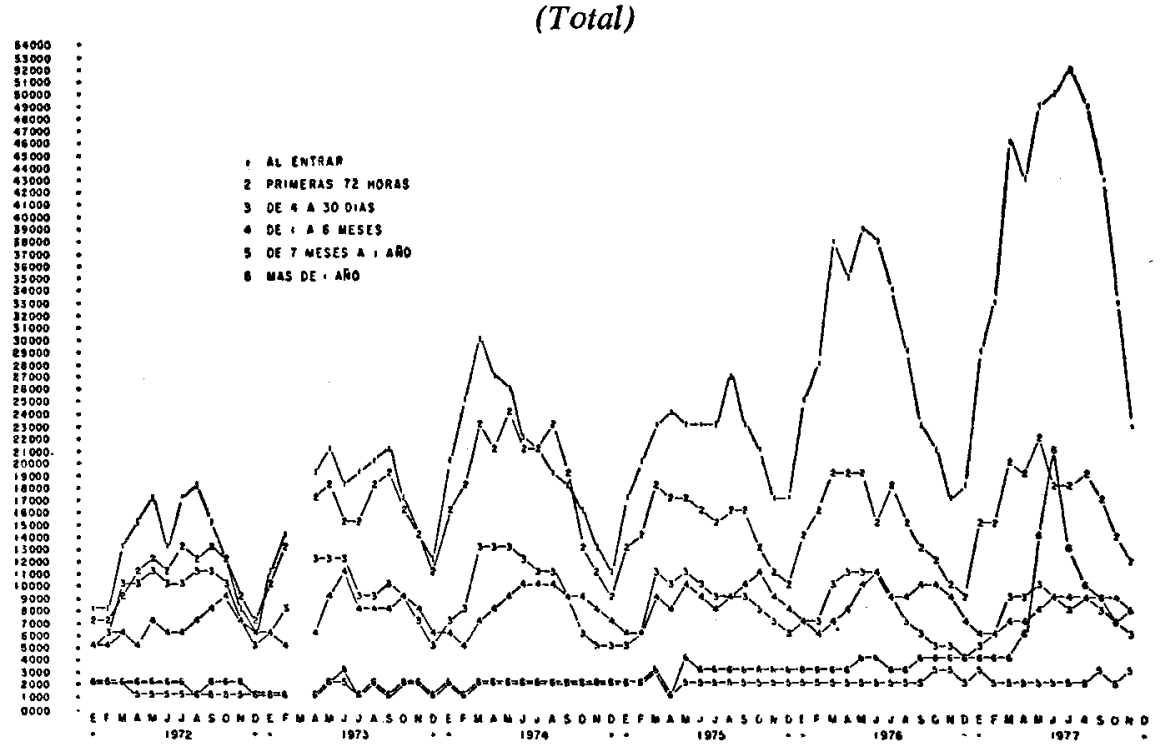

categorías de capturados antes de 72 horas desde su arribo, componen la mayoría de las expulsiones y representan el $64.04 \%$ de todas ellas. Esta corta permanencia de la mayor parte de los indocumentados que son expulsados de los Estados Unidos, puede estar relacionada con el carácter selectivo del proceso de captura, puesto que es en la zona fronteriza con México donde opera la mayor parte de los encargados de las detenciones del siN. Este hecho, junto con el notorio aumento observado sobre todo en las aprehensiones prácticamente al momento de entrar, indican obviamente una mayor actividad de la patrulla fronteriza ${ }^{11}$ al servicio del SIN.

11 Véase Julián Samora, Los mojados: The Wetback Story, Notre Dame, University of Notre Dame Press, 1971, pp. 48-51. 
La gráfica y los coeficientes obtenidos señalan en general, que los volúmenes de las capturas disminuyen conforme transcurre el tiempo de estancia. Con excepción de ciertas peculiaridades, que más adelante se comentan debido a su importancia propia, se observa que en aquellos tiempos de estancia de siete meses a un año y de más de un año, las cantidades de aprehensiones son las más bajas. A esto se añade el que las variaciones cíclicas anuales, claramente observadas en otras series cronológicas de ese fenómeno, se diluyen en estos casos de larga permanencia. En estas últimas dos categorías los índices no acusan el carácter estacional alrededor de la regresión, y sus coeficientes de variación resultan muy grandes (en algún caso de más del $80 \%$ ), lo cual indica la ausencia de un comportamiento definido. Indica además que hay más movimientos de carácter irregular. Esto último probablemente se deba a que un indocumentado que permanece dentro de los Estados Unidos por más de seis meses es un individuo que ha tenido algún éxito, en el sentido de que ha logrado internarse dentro del territorio norteamericano, ha encontrado trabajo y ha aprendido a evadir a las autoridades del sin. Más que a una rutina de patrullaje fronterizo masivo, su captura obedece a las campañas organizadas por el sIN para buscar indocumentados en áreas específicas en el interior de los Estados Unidos. ${ }^{12}$

En la gráfica 2 se observa un hecho sobresaliente. Éste se refiere al nivel que alcanzan en el año de 1977 las expulsiones de indocumentados. con más de un año de estancia antes de su detención. Registran un aumento que resulta fuera de todo lo que se esperaría conforme al crecimiento experimentado hasta antes de ese año. A tal grado resultaba perturbante dentro de la tendencia numérica de las expulsiones, que hubo necesidad de construir las rectas de regresión utilizadas para los índices de variación sólo con datos hasta marzo de 1977 inclusive. En estas condiciones, los índices de estacionalidad en los meses de mayores aprehensiones - mayo, junio y julio- son de 3.78, 5.51 y 3.26, en contraste con las medias de esos índices, las cuales son $1.63,1.95$ y 1.36 , respectivamente. Este fenómeno en particular requiere un análisis más detallado de la información.

La gráfica 3, correspondiente a las aprehensiones de hombres adultos clasificadas según el tiempo de estancia, siguen un comportamiento muy semejante al del total, con la excepción muy notoria de aquellos capturados en 1977 con más de un año de estancia. En la gráfica y en las cifras no se observa el mismo aumento relativo como en el total, a pesar de que por lo general la norma de comportamiento de las expulsiones en conjunto esté prácticamente determinada por las pautas que siguen las expulsiones de hombres adultos en virtud de su volumen: $85.80 \%$ de

12 Véase Jorge A. Bustamante, "Espaldas mojadas, informe de un observador participante", Revista de la Universidad, Vol. XXVII, № 6 (febrero de 1973), pp. 26-46. 
hombres contra $13.20 \%$ de mujeres y niños. Aun cuando es cierto que existe un aumento descomunal durante este año, no es tan acusado como el total. Los índices de estacionalidad en esos meses de mayor actividad (mayo, junio y julio) son de 2.94, 4.36 y 2.62 respectivamente, menores al valor general ya citado para esos mismos meses.

La explicación se deduce con ayuda de la gráfica 4, la que representa el mismo tipo de estadísticas, referidas ahora al caso de las mujeres y niños. En primer lugar se observa que no se siguen las mismas pautas

\section{Gráfica 3}

DEPORTACIONES SEGÚN TIEMPo dE ESTANCIA HaSta LA CAPTURA (Hombres)

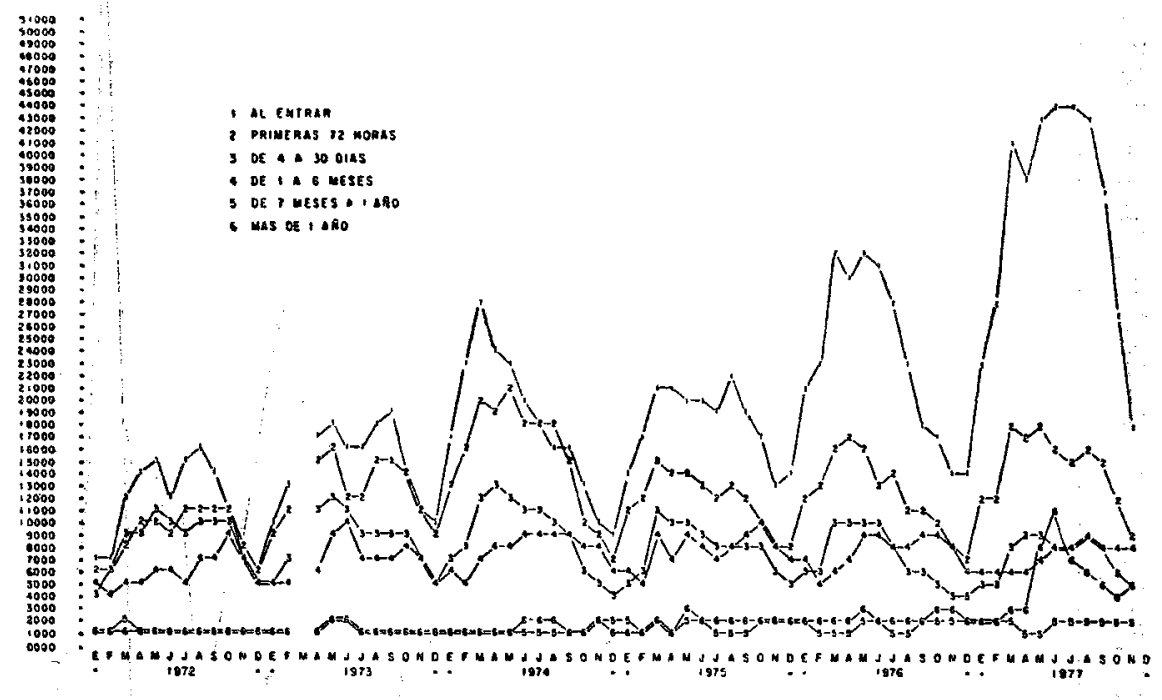

como en la gráficas anteriores. La estacionalidad anual se da sólo en aquellas dos primeras categorías de tiempo de estancia (hasta 24 horas, y de 24 a 72 horas de permanencia), aunque su carácter cíclico no es tan marcado como en los casos mencionados antes. Aun cuando los indices señalan esa variación cíclica, sus coeficientes de variación, más o menos elevados también, indican que la regularidad no es nítida. En las otras categorías tanto las gráficas como las cifras no muestran variaciones de carácter cíclico. En los índices no se detecta dicho carácter. Los tamaños de los coeficientes de variación indican la presencia más bien de irregularidades fuera de una pauta definida. Además de las consideraciones anteriores, se detecta un hecho sobresaliente en esta gráfica 4, que corresponde a los niveles que alcanzan en 1977 las cifras de mujeres y niños capturados y expulsados con más de un año de estancia. En la gráfica es notoria en particular la elevación de las cifras de esas 
Gráfica 4

DEPORTACIONES SEgúN TIEMPO dE ESTANCIA HASTA LA CAPTURA

(Mujeres y niños)

- Al imrasan

batuentis it noos

oxe t 50 ons

oe ineses.

Des of into
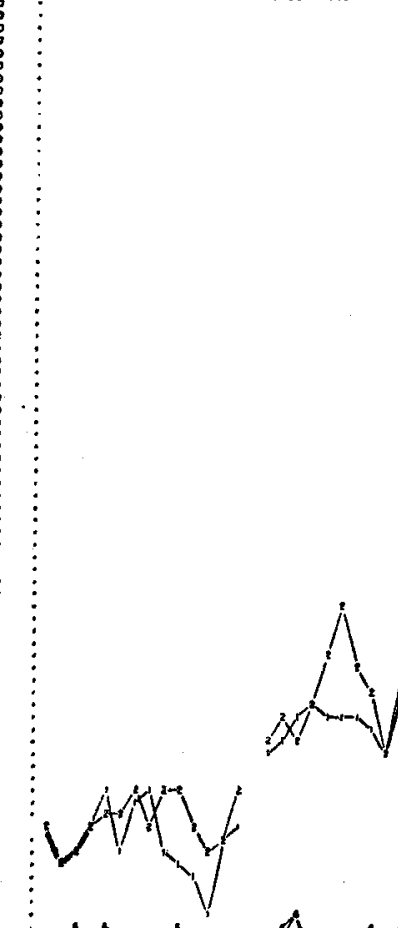

:

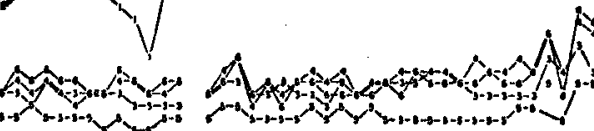


detenciones y es la que provoca que el total, representado en la gráfica 2, registre un incremento acusado a pesar de que no fuera tan notable la captura en esas condiciones en la categoría "hombres" y de que hasta antes de ese año el número de "mujeres y niños" siempre fuera relativamente menor. Las cifras de detenciones de mujeres y niños con más de un año de estancia aparecen en el cuadro 5 .

Los datos del cuadro 5, en donde se observa ese abrupto incremento de aprehensiones de mujeres y niños con más de un año de permanencia, indican que en 1977 se registraron detenciones en esa categoría en una

\section{Cuadro 5}

EXPULSIONES DE MUJERES Y NIÑOS CON MÁS DE UN AÑO DE ESTANCIA, POR MES Y AÑO

\begin{tabular}{lrrrrrr}
\hline & 1972 & 1973 & 1974 & 1975 & 1976 & 1977 \\
\hline Enero & 457 & 472 & 412 & 531 & 1046 & 1210 \\
Febrero & 428 & 458 & 388 & 587 & 950 & 1198 \\
Marzo & 629 & 56 & 434 & 877 & 1104 & 1430 \\
Abri3 & 456 & 446 & 536 & 353 & 1135 & 2285 \\
Mayo & 574 & 530 & 435 & 1956 & 1424 & 6865 \\
Junio & 511 & 704 & 579 & 1017 & 1428 & 10056 \\
Julio & 460 & 365 & 516 & 864 & 1327 & 5930 \\
Agosto & 435 & 468 & 520 & 812 & 1193 & 4545 \\
Septiembre & 434 & 307 & 528 & 846 & 1370 & 3766 \\
Octubre & 622 & 477 & 522 & 927 & 1389 & 7996 \\
Noviembre & 482 & 477 & 557 & 958 & 1537 & 3334 \\
Diciembre & 401 & 353 & 464 & 1334 & 1161 &. \\
\hline
\end{tabular}

proporción de 5 y media veces la observada en los meses de mayo, junio y julio del año anterior. En el mes de mayor número de capturas (junio) la cifra correspondiente de 1977 fue superior a 7 veces las practicadas ese año en el mismo mes.

Visto desde otro ángulo, también numérico, el fenómeno resulta igualmente notorio si se comparan los índices de estacionalidad de 1977 con la media (véase el cuadro 6). En él se observan las cifras bastante mayores del año de 1977, justamente en los meses de verano, y la perturbación que causaron en los coeficientes de variación.

En busca de explicaciones sobre ese suceso notable, se encontraron algunos informes casuísticos, elaborados de manera esporádica en ese año de $1977,{ }^{13}$ los cuales dan cabida a sospechar que las autoridades del

13 Migration Today, Vol. VI, No 3 (junio de 1978), p. 24. Por otra parte consultamos a algunos estudiosos de la materia respecto de posibles explicaciones del aumento repentino de aprehensiones de mujeres y niños que muestra la gráfica 4. En estas consultas surgió la referencia al caso Silva vs. Levi, mismo que produjo efectos de suspensión de la expulsión de indocumentados mexicanos. La posibilidad de que la gráfica mostrara una presentación voluntaria del indocumentado al siN para obtener los beneficios del caso Silva quedó descartada con la declaración que hiciera el Sr. Leonel J. Castillo, director del sIN, en audiencia pública 
SIN habían lanzado una campaña para detener y expulsar a los indocumentados "permanentes" y reducir el número de hijos de indocumentados expulsando en particular a las mujeres de mayor tiempo de estancia ilegal en los Estados Unidos. Al margen de la conexión que exista entre tales informes esporádicos y un supuesto plan cuyo objetivo fuera la aprehensión masiva de mujeres y niños, con algún grado de arraigo

\section{Cuadro 6}

COMPARACIÓN DE LOS ÍNDICES DE ESTACIONALIDAd ENTRE 1977 Y EL NÚMERo MEDIO MENSUAL DE EXPULSIONES DE MUJERES Y NIÑ̃OS CON MÁS DE UN AÑO DE ESTANCIA

\begin{tabular}{|c|c|c|c|}
\hline & 1977 & Media & $\mathrm{cv}^{2 /}$ \\
\hline $\begin{array}{l}\text { Enero } \\
\text { Febrero } \\
\text { Marzo } \\
\text { Abril } \\
\text { Mayo } \\
\text { Junio } \\
\text { Julio } \\
\text { Agosto } \\
\text { Septiembre } \\
\text { Octubre } \\
\text { Noviembre } \\
\text { Diciembre }\end{array}$ & $\begin{array}{l}1.0037 \\
0.9805 \\
1.2358 \\
1.8217 \\
5.4032 \\
7.8147 \\
4.5608 \\
3.4450 \\
2.8198 \\
2.2163 \\
2.4370 \\
\text { No disponible }\end{array}$ & $\begin{array}{l}1.0750 \\
0.9992 \\
1.2776 \\
1.1131 \\
1.9312 \\
2.3518 \\
1.5859 \\
1.3702 \\
1.2295 \\
1.2570 \\
1.2462 \\
0.8525\end{array}$ & $\begin{array}{r}41.3466 \\
36.2977 \\
46.0675 \\
43.3891 \\
82.8584 \\
104.4132 \\
85.0783 \\
68.8997 \\
61.0141 \\
42.0543 \\
45.9613 \\
26.7755\end{array}$ \\
\hline
\end{tabular}

a Coeficientes de variación.

en territorio de los Estados Unidos, tanto gráficas como cifras demuestran que en 1977 tuvo lugar una acción de captura fuera de lo común, y que en particular estuvo orientada a la búsqueda y expulsión de mujeres y niños con un tiempo prolongado de estancia dentro de los Estados Unidos. Es necesario destacar que la fuente de datos que sustentan estos hechos descartan la posibilidad de que la detención y consecuente expul. sión de mujeres y niños haya sido en ese año un mero producto del azar o de un aumento repentino de la migración de mujeres y niños, puesto que es precisamente en la clasificación de más de un año de estancia donde se da el incremento y no en las categorías de menor tiempo de estancia.

ante el Comité Legislativo de la Cámara de Diputados de los Estados Unidos, el 8 de febrero de 1978, en la que dijo lo siguiente: "On the basis of the June 21 , 1977 order in the Zambrano case, the Service and the Department of State began processing applications for adjustment of status and for immigrant visas filed by members of the Silva class" (Con base en la orden judicial del 21 de junio de 1977 en el caso Zambrano el Servicio y el Departamento de Estado empezaron a proceder solicitudes para ajuste del status y para visas de inmigrante presentados por los miembros de la clase Silva). La fecha señalada por Castillo es varios meses posterior al inicio de las aprehensiones a las que se refiere la gráfica 4 . 
La gran diferencia numérica en la categoría de expulsiones discutida demuestra una acción selectiva en las aprehensiones realizadas en 1977, evidencia de que hubo una decisión de las autoridades migratorias estadounidenses para realizar una operación masiva de expulsiones, en particular aquellas mujeres y niños con una permanencia en el país mayor a un año. Estas expulsiones se llevaron a cabo justamente en los meses en los cuales se produjeron declaraciones por parte de altos funcionarios del gobierno federal de los Estados Unidos, incluido el propio presidente, ${ }^{14}$ en las cuales se negaba todo propósito de llevar a cabo expulsiones masivas y se ponían de relieve las intenciones del gobierno norte-

\section{Cuadro 7}

EXPULSIONES DE INDOCUMENTADOS CON MÁS DE UN AÑO DE ESTANCIA EN 1977, POR MESES

\begin{tabular}{|c|c|c|}
\hline & Hombres & Mujeres y ninos \\
\hline $\begin{array}{l}\text { Enero } \\
\text { febrero }\end{array}$ & $\begin{array}{ll}2 & 465 \\
2 & 406\end{array}$ & $\begin{array}{ll}1 & 210 \\
1 & 198\end{array}$ \\
\hline narzo & 2879 & 1530 \\
\hline abril & 3297 & 2285 \\
\hline Nayo & 7542 & 6865 \\
\hline Janio & 11206 & $10 \quad 056$ \\
\hline Jutio $=$ & 6803 & 5930 \\
\hline ngosto & 5502 & 4345 \\
\hline Sepriemore & 4918 & 3766 \\
\hline octubre & 4265 & 2996 \\
\hline Novieabre & 4937 & 3334 \\
\hline
\end{tabular}

americano de respetar los derechos humanos de los indocumentados mexicanos, dentro de los cuales se encuentra el derecho a la unidad familiar.

La notoriedad de esa clase particular de expulsiones de gentes indocumentadas con más de un año de permanencia, sugiere que las autoridades del sin llegaron a la decisión de expulsar a indocumentados, posiblemente en familias, que pudieran ser eventualmente beneficiadas por la propuesta de amnistía que ya en la primera mitad de 1977 se conocía que haría la presidencia. Esto se sustenta en el hecho del gran parecido que existe entre las cifras absolutas de expulsados en esas categorías de más de un año de estancia tanto para hombres como para mujeres y niños. Esta semejanza se evidencia al comparar las cifras absolutas en ambas categorías para 1977, tal y como se presentan en el cuadro 7.

\section{LAS EXPULSIONES Y LA CONDICIÓN LABORAL}

En las gráficas 5,6 y 7 se observa el comportamiento de las deportaciones de acuerdo con la clase de actividad de los indocumentados en

14 Jorge A. Bustamante, "Las propuestas de política migratoria en los Estados Unidos y sus repercusiones en México", Foro Internacional, Vol. XVIII, No 3, El Colegio de México, 1978. 
Gráfica 5

DEPORTACIONES SEGÚN CONDICIÓN LABORAL

(Total)

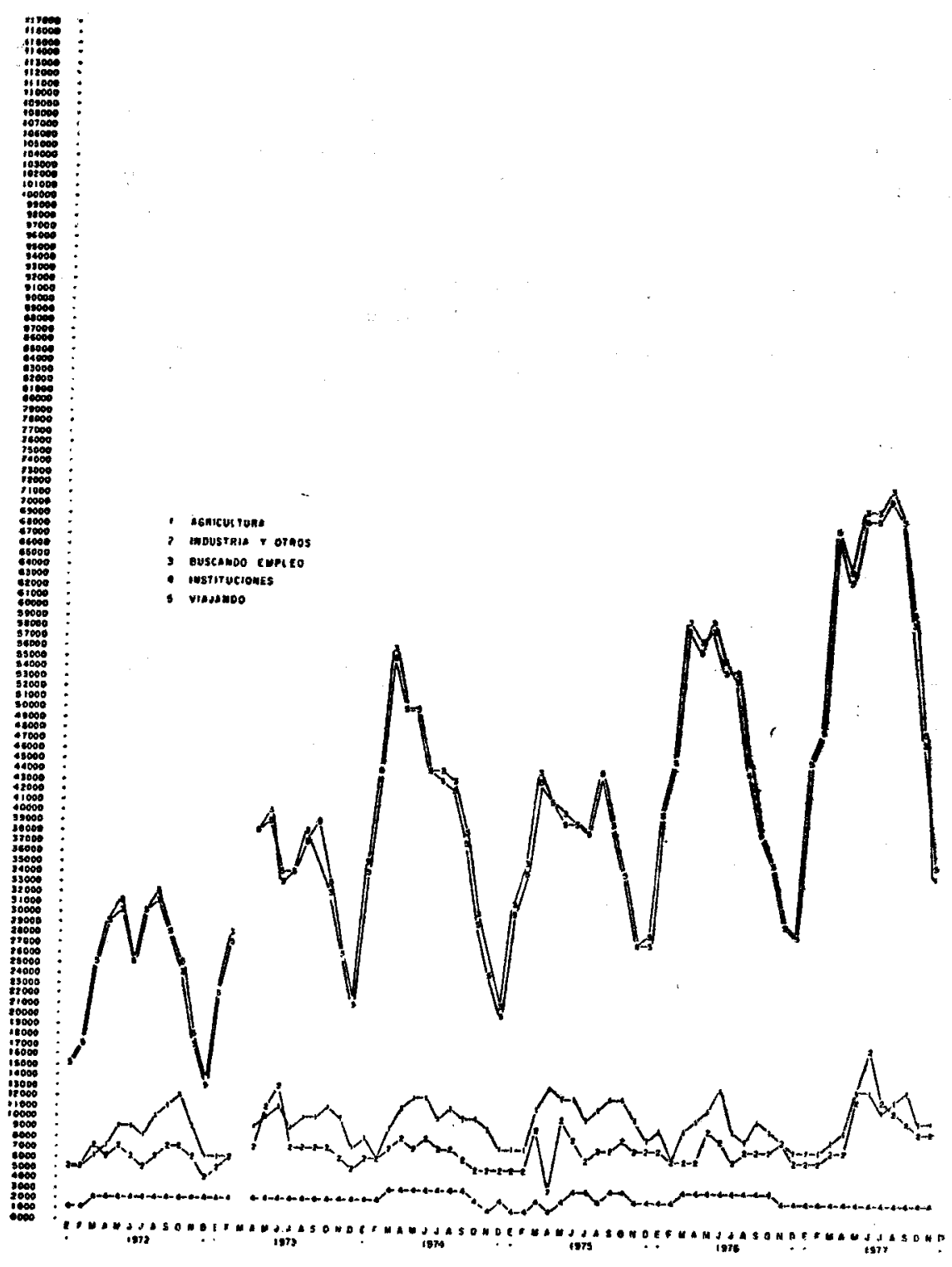


el momento de su captura. En la gráfica 5 se resume la totalidad de las expulsiones; la 6 se refiere a la de hombres adultos y la 7 a las deportaciones de mujeres y niños.

En la gráfica 5 se aprecia la existencia del ciclo anual observado desde un principio, y lo mismo sucede con la gráfica 6 que corresponde a hombres adultos. En ambos casos este ciclo anual se perfila de manera notoria en las clasificaciones que corresponden a los indocumentados que fueron detenidos mientras viajaban o buscaban empleo. Aun cuando el ciclo no es tan manifiesto también se detecta para los que estaban empleados en la agricultura o en la industria y otras actividades. Donde no se registra un comportamiento cíclico es en aquellas expulsiones que corresponden a los migrantes identificados como indocumentados mientras se encontraban en alguna institución como hospitales o cárceles. Aparte de estas particularidades, en las tres gráficas de estas clasificaciones es notoria la gran coincidencia y el gran volumen que forman aquellos contingentes de indocumentados de ambos sexos y todas las edades que se encuentran viajando y buscando empleo. Esta correspondencia se debe al hecho de que son precisamente los buscadores de empleo los que se encuentran en tránsito. Al mismo tiempo debe haber una alta correlación entre este tipo de indocumentados y su tiempo de permanencia en los Estados Unidos, con toda seguridad de carácter muy corto. (Una manera de apreciar esto último es a través de las regresiones lineales que resultan de cada clasificación. Tanto para el total de indocumentados cuya captura se hizo en las primeras 72 horas, como para aquellos capturados mientras buscaban empleo y para los detenidos mienras viajaban, las regresiones resultaron con coeficientes prácticamente iguales).

En lo que respecta a los buscadores de empleo y de gentes en tránsito que fueron detectados y detenidos como indocumentados (véanse las gráficas 5 y 6), se manifiesta con mayor nitidez la disminución registrada en 1975 a la cual se hizo referencia cuando se discutieron los totales mostrados en la clasificación correspondiente a la gráfica 1. Esto refuerza la hipótesis ya citada de que el aumento del desempleo en los Estados Unidos tuvo un efecto directo en el volumen del flujo de inmigrantes indocumentados buscadores de empleo. Estas migraciones indocumentadas tienen prácticamente como único motivo la consecusión de medios de vida, lo cual explica esa correspondencia entre las fluctuaciones de la demanda de mano de obra y el volumen de migrantes mexicanos sin documentos hacia los Estados Unidos.

Tanto en la gráfica 5 como en la 6 se observa que el número de expulsados empleados en la agricultura es mayor que el de aquellos in. volucrados en actividades industriales y de otro tipo. A su vez, ambas categorías son mayor que las expulsiones de indocumentados que fueron localizados en instituciones. Hay dos excepciones, una en el segundo tri- 
Gráfica 6

DePORTACIONES SEgúN CONDICIÓN LABORAL (Hombres)

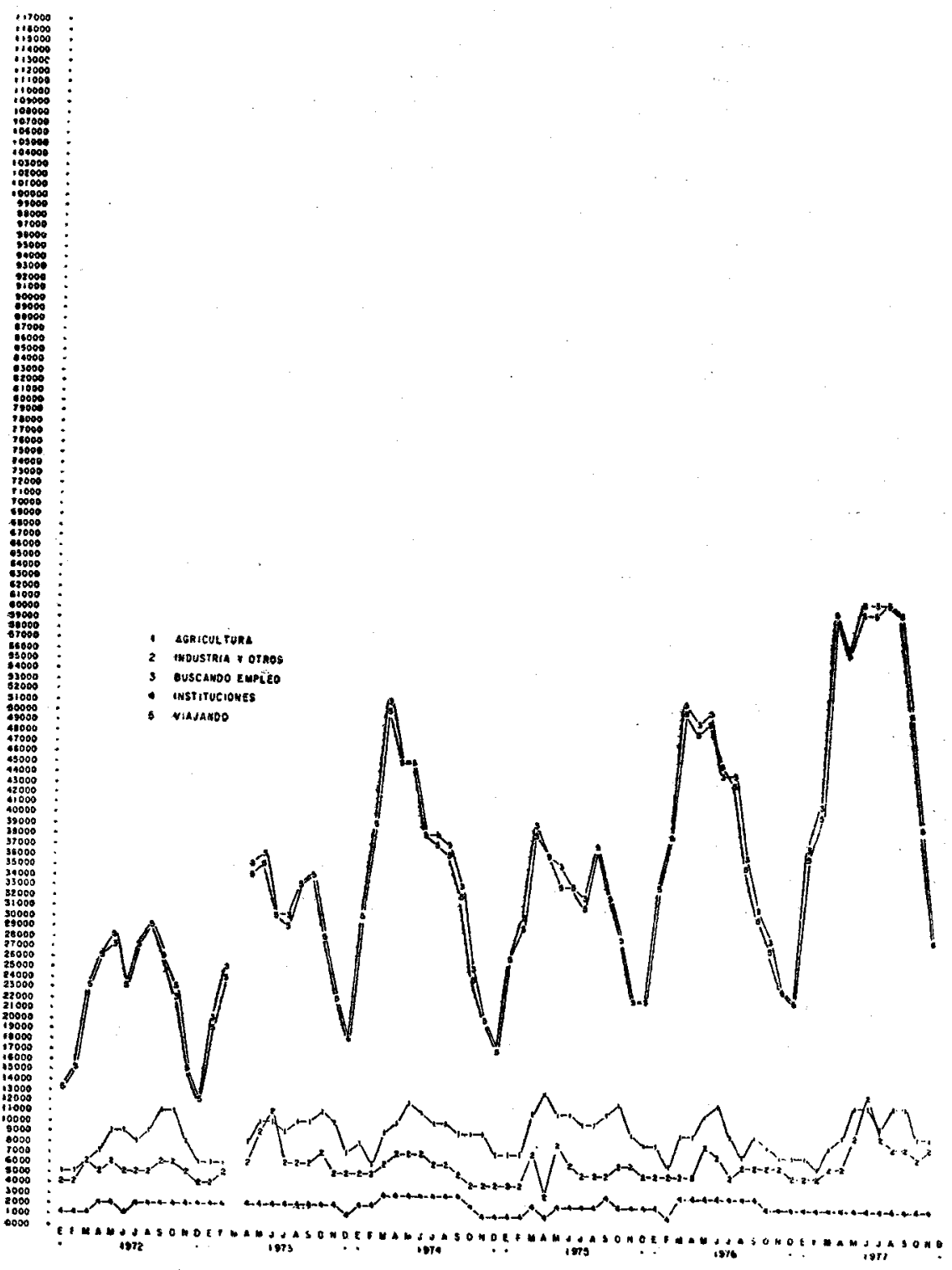


mestre de 1973 y la otra a mitad de 1977. En ambos momentos la explicación debe estribar en la existencia de alguna campaña dirigida a la captura de indocumentados cuyas actividades se pueden clasificar en la rama de "industria y otros".

Al analizar la gráfica 7 , la cual se refiere a las mismas clasificaciones de condición laboral, es también notorio el carácter creciente y cíclico anual de aquellos indocumentados que se encontraban viajando buscando empleo al ser detenidos, y casi con los mismos volúmenes en ambas categorías. La misma gráfica permite también observar que en este caso

\section{Gráfica 7}

DEPORTACIONES SEGÚN CONDICIÓN LABORAL

(Mujeres y niñas)

1. agriculitura

a industria r OtRos

- instituciones

5 viasanoo

los dedicados a "industrias y otros" representan mayor número de expulsiones que los empleados en actividades agrícolas. Además, los volúmenes expulsados de ambas categorías resultan mayores respecto a los aprehendidos que se encontraban en instituciones. Este último resultado puede ser explicado por la circunstancia de que es menor el número de mujeres que se emplean en actividades del campo, y a que la categoría de "industria y otros" incluye a las trabajadoras en servicios domésticos, así como a las que simplemente son amas de casa y a los niños. De cualquier manera, el hecho más notable observado en esta gráfica es el gran aumento de expulsiones logradas por el siN en 1977, justamente en esa categorización de mujeres y niños en "industrias y otros". En los meses 
Gráfica 8

DEPORTACIONES SEGÚN SITUACIÓN MIGRATORIA

andigandu

(Total)

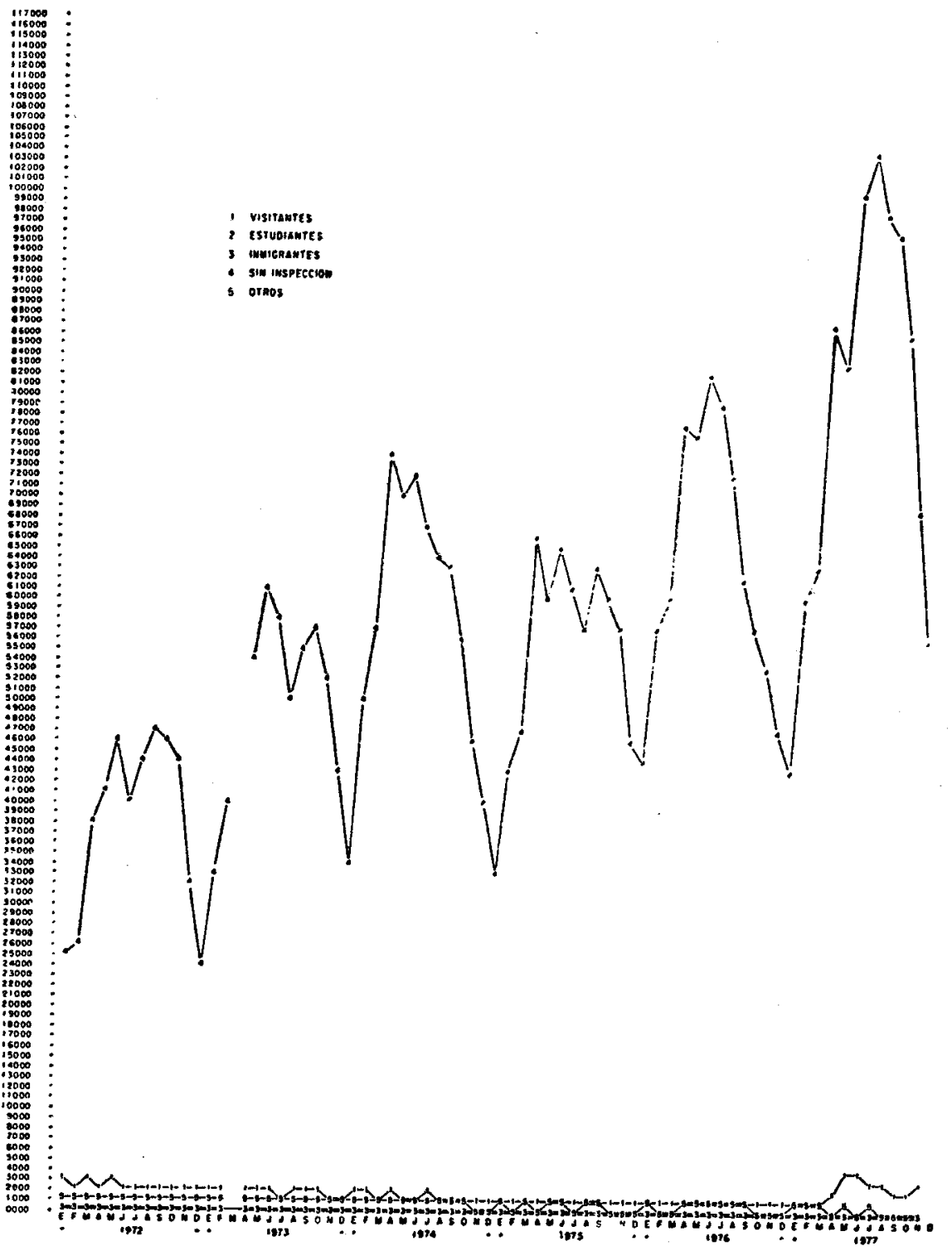


de mayo a septiembre se hicieron expulsiones de ese tipo en mucho más del doble de la cantidad que se hubiera esperado en condiciones "normales". Esto corrobora las deducciones hechas en relación con la gráfica 4.

Sobre esta serie de datos, clasificados por condición laboral, cabe destacar que la notable disminución de capturas registrada en 1975, se manifiesta de manera particular en las categorías de viajantes y buscadores de empleo, junto con un dato específico que se refiere a una disminución de las capturas en la categoría de "industrias y otros" en el mes de abril de ese mismo año de 1975. Este hecho pudo ser puramente casuístico dentro de las actividades policíacas del siN.

\section{LA SITUACIÓN MIGRATORIA DE LOS EXPULSADOS}

La gráfica 8 muestra la preponderancia de la categoría de "entradas sịn inspección". ${ }^{15}$ Esta clasificación corresponde a los migrantes indocumentados que entraron dentro del territorio norteamericano sin ningún tipo de documento, lejos de los puestos de autorización migratoria, categoría que constituye la mayoría de los indocumentados. El $96 \%$ de las expulsiones se dan en este tipo de indocumentados, quienes por lo general, son los que vadean el río durante la noche, o atraviesan la frontera en algún lugar desértico.

El resto de las categorías a las que se hace referencia en la gráfica 8 , se refieren a aquellos indocumentados que entraron a los Estados Unidos con algún tipo de documento migratorio que les permitía una estancia limitada y no autorizados a obtener un empleo en aquel país. Al excederse en los términos de las visas o procurar un empleo, se colocaron automáticamente en la categoría de indocumentados. La gráfica 8 muestra la distinción entre las pautas de expulsión correspondiente a los que entraron sin ningún documento. Se aprecia con claridad la correspondencia entre los de la primera categoría con el comportamiento cíclico general observado en las gráficas comentadas anteriormente. Por último, cabe observar que el aumento de las aprehensiones en el año 1977, correspondiente a la categoría de "visitantes", refleja una vez más los efectos de una campaña específica de expulsiones que afectó a los indocumentados en su estructura familiar y de tiempo de estancia.

15. Entry without inspection (EWI). 